\title{
Pedicled thoracodorsal artery perforator flap for reconstruction of post burn contracted neck
}

\author{
Osama Alshahhat, *Mohamed Autifi, Mohamed Hosny, Mohamed Osama, Mohamed Farid \\ and Moaaz Moaty \\ Departments of Plastic Surgery and *Anatomy and Emberyology, Faculty of Medicine, Al-Azhar \\ University
}

Corresponding author: Moaaz Mahmoud Abd Almoaaty, E-Mail: moaazplastic@azhar.edu.eg, Mobile: 01126223879

\begin{abstract}
Background: The thin and pliable skin of the neck is a region with multidirectional activity, and postburn contractures forms there easily. Different methods of reconstruction were described. In our study the thoracodorsal artery perforator flap (TDAP) is used to reconstruct the post burn contracted (PBC) neck. Its main vascular supply is the thoracodorsal artery, and can be harvested as an island flap. Aim of the work: to evaluate the aesthetic and function outcome of TDAP flap as a new technique for reconstruction of PBC neck.

Patients and Methods: this study included a total of 10 TDAP flaps. Were studied prospectively for their efficacy in reconstruction of PBC neck in 10 patients. Also donor site morbidity, patient satisfaction, and complications were evaluated. Between November of 2017 and January of 2019, this study was preceded by preserved cadaveric dissection. All were island flaps. The range of flap size was $18 \pm 5 \mathrm{~cm}$ in length, and $9 \pm 2 \mathrm{~cm}$ in width. Primary closure of donor site was performed in all cases.

Results: 8 of the 10 flaps survived completely, two cases of distal necrosis not exceeding $2 \mathrm{~cm}(20 \%)$, and no total flap necrosis. Most of the patients were satisfied with both the functional and aesthetic results.

Conclusion: PBC neck affects function, and appearance. In our view, the island TDAP flap is a good option for neck resurfacing regarding a wide arc of rotation, a relatively larger flap dimensions, a reliable pedicle length, and acceptable donor site morbidity.
\end{abstract}

Keywords:Thoracodorsal artery perforator flap, post-burn neck contracture, island flap, fasciocutaneous flap.

\section{Introduction}

Post-burn neck contractures are a challenging problem for plastic surgeons. Post burn contracted neck is characterized by restrictions in range of neck motion, especially extension. Functional and cosmetic abnormalities resulting from this type of distortion can cause extreme depression ${ }^{(\mathbf{1})}$.

In 2005 Onah classified the burn contractures of the neck based on the numeric categories of 1 to 4 (type 1-type 4), which include position, severity, and likely problems. Subgroups within each numeric category are used to designate the width of the contracture, which affect the options available for reconstruction. The numeric category depends on the extent of flexion or extension by the contracted neck and the anatomical position of the neck ${ }^{(2)}$.

The post burn contracted neck can be reconstructed with a wide range of surgical techniques, including skin grafts, pre-expanded flaps $^{(3)}$, and perforator flaps ${ }^{(4)}$.

Based upon the use of TDAP flap for hypopharyngeal reconstruction and its reachability to the neck ${ }^{(5)}$ we used it in neck resurfacing.

The purpose of this article is to report our clinical experience with the use of island TDAP flap as a new reconstructive modality for neck re-surfacing. We aim to emphasize the surgical technique, postoperative complications, and the functional outcome.

\section{Aim of the study}

The aim of the study is to evaluate the aesthetic and function outcome of thoracodorsal artery perforator flap as a new technique for reconstruction of post burn contracted neck.

\section{Patients and Methods}


This study was carried out in the Plastic, Burn and Reconstructive Surgery and Anatomy Departments of Al-Azhar University between November 2017 and January 2019. The study was approved by the Ethics Board of AlAzhar University.

Preserved cadaveric dissections of the flap (three cadavers) that were done in the Morgue of Al-Azhar Faculty of medicine.

Clinical study involved 10 patients ( 6 females and 4 males) who were suffering from postburn neck contracture, and who were subjected to reconstruction with 10 unilateral island thoracodorsal artery perforator flaps. Age ranged from 10 to 54 years. 4 cases were of type Ic and 6 cases of type Пc). We harvested 5 flaps from the right side and 5 from the left. The size of the reconstructed defect ranged from $23 \times 10$ to $14 \times 6$, and the dimensions of the flap varied from $16 \times 8 \mathrm{~cm}$ to $22 \times 7 \mathrm{~cm}$. Follow-up period ranged from 4 to 12 months (average 6.3).

Operative steps: After intubation, while the patient is in the supine position with hyperextension of the neck (by putting a pillow underneath the upper back). Neck contracture release was done till hyperextension was obtained.

Patient repositioning: After neck contracture release, the patient was installed in the lateral position either right or left. The ipsilateral shoulder was abducted $90^{\circ}$ and the elbow was flexed $60^{\circ}$ to $90^{\circ}$, and the forearm was installed on a side arm. The ipsilateral upper limb and the back were prepared and draped.

Flap Design: The design of the flap is based on the dimensions and the orientation of the defect. Based on the previous anatomic studies, the TDAP flap perforators arise from the lateral branch of the thoracodorsal artery ${ }^{(6)}$.

Preoperative perforator mapping was performed with a unidirectional Doppler probe. The perforators were found in a region $8 \mathrm{~cm}$ below the posterior axillary fold and $2 \mathrm{~cm}$ behind the anterior border of the L.D. muscle. The flap was designed in a vertical orientation with its anterior border extending beyond the anterior border of the muscle.

Flap Dissection: Skin incision started at the anterior border of the flap. The incision was deepened to reach the deep fascia of the serratus anterior muscle. Dissection then continued to reach the anterior border of the latissimus dorsi muscle. The anterior border of the muscle was retracted laterally to identify the underlying lateral branch of the thoracodorsal vessels. The lateral branch was traced superiorly until it reached the level of bifurcation of the thoracodorsal vessels. The flap perforators could then be visualized originating from the lateral branch just below the bifurcation. The inferior portion of the flap was then elevated to the region of the perforators.

Thoracodorsal Pedicle Dissection: Dissection of the flap perforators continued with the cuff of muscle around them to the level of the bifurcation of the thoracodorsal vessels. The flap was then freed from the rest of the muscle through widening the split in the muscle fibers to allow the flap to pass through that split. Ligation of the medial branch of the thoracodorsal vessels was performed at the level of bifurcation. Dissection continues over the pedicle with great caution to avoid injuring the thoracodorsal nerve. Ligation of the serratus anterior muscle branch and the circumflex scapular vessels was performed and the dissection of the subscapular vessels continued until the axillary vessels were reached.

Subcutaneous tunnel for the flap: A subcutaneous tunnel for the flap was created by blunt finger dissection. Dissection continued with bimanual assistance of the other hand. The tunnel was widened to accommodate the passage of at least two fingers. Long artery forceps was introduced cautiously through the tunnel from the neck to reach the axilla and hold the flap. The flap was pulled through the tunnel to reach the neck and was then passed

Closure of the donor site: Primary closure in two layers (subcutaneous tissue and skin) was the goal to limit the donor site morbidity; a suction drain was put on the surface of the LD muscle.

Flap insetting: The flap was sutured in place using interrupted sutures.

Statistical methods:

Data entry and statistical analyses were performed using SPSS (statistical package of social sciences) version 21 (SPSS Inc., Chicago, IL, USA). Categorical data were expressed in number and percentage. Continuous normally distributed data were expressed in mean and standard deviation.

Independent sample $\mathrm{T}$ test was used for continuous normally distributed data. To study the association between Categorical data chi square test was used. Statistical significance 
was considered when probability $(\mathrm{P})$ value was less than or equal to 0.05 .

\section{Results: table I and II}

This study was conducted for 10 patients, 6 females and 4 males, with an average age of 28 years. They had post-burn neck contractures (4 cases of grade Ic and 6 cases of grade $\Pi c)$. All cases were reconstructed with a TDAP flap. We harvested 5 flaps from the right side and five from the left.

Donor site was closed primarily in all cases. We harvested flaps of various lengths according to the expected defects after excision of cicatrizing tissue. Flap length ranged from a minimum of $16 \mathrm{~cm}$ to a maximum of $25 \mathrm{~cm}$. Mean length was $21.7 \mathrm{~cm}$, with a standard deviation of 2.52 (Table I).

The width of the flaps harvested ranged from a minimum of $7 \mathrm{~cm}$ to a maximum of $11 \mathrm{~cm}$. Mean width was $9.7 \mathrm{~cm}$, with a standard deviation of 1.17 (Table I).

The flap pedicle length ranged from $15 \mathrm{~cm}$ to $23 \mathrm{~cm}$ mean length was $18.8 \mathrm{~cm}$.
In our study, the harvested flaps had a relatively large surface area, ranging from a minimum of 144 to a maximum of 275 with a mean of 211.6 $\mathrm{cm} 2$ and a standard deviation of 42.2 (Table I).

The following complications occurred: hematoma in 2 cases (20\%), partial distal necrosis in 2 cases, no total flap necrosis and disruption of the wound in 2 cases. As regards donor site complications, hypertrophic scars occurred in 2 cases and 4 cases of widened scars (40\%) in which the donor site was closed with split thickness graft (Stsg) due to flap width exceeding $10 \mathrm{~cm}$.

The esthetic quality of the neck was judged by the patient, operating team, and patient's relative. It was found esthetically fair to good

The total operative time ranged from 150 to 210 minutes with the mean time 171 minutes.

All patients resumed their shoulder active range of motion within 3 weeks postoperatively with only limited active forward movement.

\section{Table (1): demographic characteristic of the study}

\begin{tabular}{|c|c|c|c|c|c|c|c|c|}
\hline Case & Age & Sex & $\begin{array}{c}\text { Contractur } \\
\text { e type }\end{array}$ & Side & Defect size & $\begin{array}{l}\text { Flap size } \\
(\mathrm{cm})\end{array}$ & Pedicle length & $\begin{array}{c}\text { Donor site } \\
\text { closure }\end{array}$ \\
\hline (1) & $23 \mathrm{y}$ & F & IIc & Rt. & $19 \times 8$ & $21 \times 9$ & $19 \mathrm{~cm}$ & 1ry \\
\hline (2) & $33 \mathrm{y}$ & $\mathrm{M}$ & Ic & $\mathrm{Lt}$ & $21 \times 10$ & $23 \times 11$ & $22 \mathrm{~cm}$ & 1ry \\
\hline (3) & $18 \mathrm{y}$ & $\mathrm{F}$ & IIc & $\mathrm{Rt}$ & $20 \times 6$ & $22 \times 7$ & $17 \mathrm{~cm}$ & 1ry \\
\hline (4) & $23 \mathrm{y}$ & M & Ic & $\mathrm{Lt}$ & $21 \times 8$ & $23 \times 10$ & $21 \mathrm{~cm}$ & 1ry \\
\hline (5) & $10 y$ & $\mathrm{~F}$ & IIc & Lt. & $16 \times 8$ & $20 \times 10$ & $15 \mathrm{~cm}$ & 1ry \\
\hline (6) & $19 \mathrm{y}$ & $\mathrm{F}$ & IIc & $\mathrm{Lt}$ & $21 \times 10$ & $23 \times 11$ & $16 \mathrm{~cm}$ & 1ry \\
\hline (7) & $15 \mathrm{y}$ & M & Ic & Rt. & $20 \times 7$ & $22 \times 9$ & $18 \mathrm{~cm}$ & 1ry \\
\hline (8) & $32 \mathrm{y}$ & $\mathrm{F}$ & IIc & Rt. & $18 \times 8$ & $21 \times 9$ & $20 \mathrm{~cm}$ & 1ry \\
\hline (9) & $54 \mathrm{y}$ & M & Ic & $\mathrm{Lt}$ & $22 \times 7$ & $24 \times 8$ & $23 \mathrm{~cm}$ & 1ry \\
\hline (10) & $30 \mathrm{y}$ & $\mathrm{F}$ & IIc & Rt. & $19 \times 10$ & $21 \times 11$ & $17 \mathrm{~cm}$ & 1ry \\
\hline Mean & & & & & & & $18.8 \mathrm{~cm}$ & \\
\hline
\end{tabular}

Table (1I): demographic characteristic of the study

\begin{tabular}{|c|c|c|c|c|c|} 
Case & Flap type & $\begin{array}{c}\text { Operative time } \\
\text { (min.) }\end{array}$ & Flap complication & $\begin{array}{c}\text { Follow up } \\
\text { ( months })\end{array}$ & donor sites complications \\
\hline
\end{tabular}


Osama Alshahhat et al.

\begin{tabular}{|c|c|c|c|c|c|}
\hline$(1)$ & Island & 200 & Flap congestion & 12 & \multicolumn{2}{|c|}{ Hypertrophic scars } \\
\hline$(2)$ & Island & 210 & & 11 & \\
\hline$(3)$ & Island & 170 & & 12 & Hypertrophic scars \\
\hline$(4)$ & Island & 180 & & 9 & \\
\hline$(5)$ & Island & 150 & $\begin{array}{c}\text { partial distal } \\
\text { necrosis }(2 \mathrm{~cm} .)\end{array}$ & 6 & \\
\hline$(6)$ & Island & 160 & & 4 & \\
\hline$(7)$ & Island & 150 & $\begin{array}{c}\text { partial distal } \\
\text { necrosis }(2 \mathrm{~cm} .)\end{array}$ & 6 & Wound dehiscence \\
\hline$(8)$ & Island & 150 & Flap congestion & 5 & \\
\hline$(9)$ & Island & 180 & & 4 & \\
\hline$(10)$ & Island & 160 & & & \\
\hline Mean & & 171 & & 5 & \\
\hline
\end{tabular}

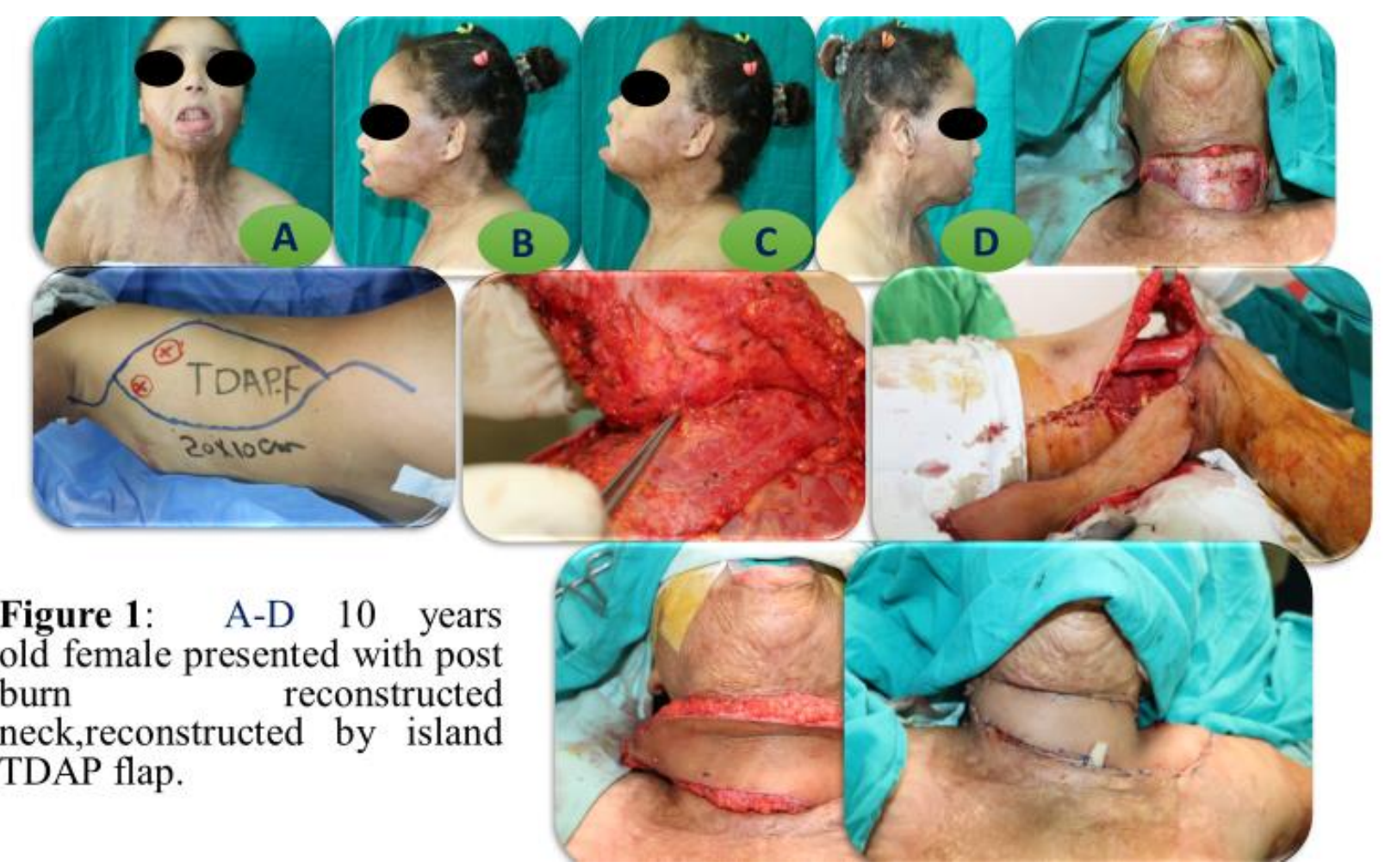

\section{Discussion}

Patients with post-burn contracted neck usually exhibit severe restriction of neck movement and related deformities such as shoulders stiffness, kyphosis posture, cicatricial ectropion of the lower lip, and incomplete oral occlusion with saliva drooling. Therefore, post burn neck contractures represent a challenge with a unique set of problems for the reconstructive surgeon, compared with the other parts of the body ${ }^{(7)}$.
Multiple reconstructive options are available for the treatment of postburn scar contractures. All of them have their advantages, disadvantages, and limitations. To obtain good results, the surgical procedure should fulfill the cosmetic (symmetry, contour, color, and texture match) and functional (mobility and facial expression) criteria of the neck ${ }^{(8)}$. A skin graft is the simplest reconstructive method but it has several disadvantages. The cervical area in the flexion position makes a graft contracture easier. Also, a skin graft is not suitable for 
coverage of exposed vital structures after release of the contractures. The recurrence is common because of the secondary skin graft contracture, especially with split-thickness skin grafts.

Local random flaps like transposition or advancement flaps are not effective in severe cervical contractures because they are often of an inadequate size. Myocutaneous flaps (e.g., pectoralis major, trapezius, and latissimus) are a good option for reconstruction of postburn neck contractures, but their bulkiness, with cervical sagging and donor-site morbidity, limits their use ${ }^{(9)}$. Free flaps are the other alternative method for coverage of cervical defects. These flaps have been successfully used for cervical defects with subsequent debulking procedures. However, free flaps are complicated and technically challenging because the burned regions limit the options of free flaps.

The trans-axillary TDAP flap is a technique used for the first time for hypopharyngeal reconstruction. It offers the advantages of free fasciocutaneous flaps, has a wide arc of rotation, a relatively larger flap dimension, a reliable vascular pedicle, and acceptable donor site morbidity ${ }^{(5)}$. Based upon this we used it for neck surfacing.

The length of the TDAP flap pedicle is a major advantage of the flap if it is used as a free or a pedicled flap. The pedicle length might reach up to $25 \mathrm{~cm}$ according to a previous study (10). The maximum pedicle length in our study was $23 \mathrm{~cm}$ and the mean length was $18.8 \mathrm{~cm}$. This has allowed comfortable passage of the flap through the subcutaneous tunnel for neck re-surfacing.

The position of the perforators may be eccentric in relation to the skin paddle. This enables proper lengthening of the pedicle (11) and this was in agreement with our study.

The flap dimensions were determined according to the defect dimensions after neck contracture release. The donor site was closed primarily in all cases. The size of the skin paddle of the flap can be as large as $25 \times 15 \mathrm{~cm}$ with the potential for direct closure of the donor

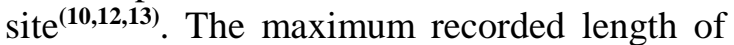
the flap in our series was $24 \mathrm{~cm}$ and the width was $11 \mathrm{~cm}$. This size allowed reconstruction of different defects of the neck burn contracture after release.

We categorized the postoperative complications in this study into flap complications and donor site morbidity.

The TDAP flap complications occurred in 4 cases (40\%) as follow: 2 cases of partial distal necrosis not exceeding $2 \mathrm{~cm}$ and 2 cases of venous congestion due to hematoma under the flap managed conservatively by stitch release and washing under the flap.

The donor site complications occurred in one case $(10 \%)$ : wound dehiscence that was managed conservatively.

The donor site scar related problems occurred in 2 cases (20\%) hypertrophic scars and 3 cases of widened scars (30\%) that may be attributed to wound closure without anchoring sutures.

The thoracodorsal nerve was routinely preserved in our series. This maintains the contour of the back, less pain in the postoperative period, and less seroma formation as compared with the standard latissimus dorsi flap ${ }^{\mathbf{( 1 0 )}}$. There were no reported contour irregularities or seromas in our series postoperatively. In 2004, Hamdi et al, assessed shoulder function after harvesting thoracodorsal artery perforator flap for breast reconstruction. ${ }^{(12)}$. Ttey recorded the same shoulder mobility to the opposite side (nonoperated) site, but both active and passive forward elevations were reduced postoperatively. Similar findings were recorded in our study.

\section{Conclusion}

Scarring of the neck produces problems with function, and appearance. In our view, the pedicled TDAP flap is an ideal technique for neck resurfacing. It offers the advantages of free fasciocutaneous flaps, has a wide arc of rotation, a relatively larger flap dimension, a reliable vascular pedicle, and acceptable donor site morbidity.

\section{References:}

1. Pallua $\mathbf{N}$, Künsebeck $H$, Noah $E$ (2003):Psychosocial Adjustments 5 Years after Burn Injury.Burns, 29(2): 143-52.

2. Onah II (2005): A classification system for postburn mentosternal contractures. Archives of Surgery, 140(7):671-675.

3. Ninkovic $M$, Moser-Rumer A, Ninkovic M, Spanio S, Rainer C, Gurunluoglu R (2004): Anterior neck reconstruction with pre-expanded free 
groin and scapular flaps. Plastic and reconstructive surgery, 113(1):61-68.

4. Ogawa R, Murakami M, Vinh VQ, Hyakusoku H (2006): Clinical and anatomical study of superficial cervical artery flaps: Retrospective study of reconstructions with 41 flaps and the feasibility of harvesting them as perforator flaps. Plastic and reconstructive surgery, 118(1):95-101.

5. Amin AAW, Rifaat M, Ellabban MA, Zedan M, Kamel M, Bassiouny M (2014): Transaxillary thoracodorsal artery perforator flap: a versatile new technique for hypopharyngeal reconstruction. Journal of reconstructive microsurgery, 30(06):397-404.

6. Heitmann C, Guerra A, Metzinger SW, Levin LS, Allen RJ(2003): The thoracodorsal artery perforator flap: anatomic basis and clinical application. Annals of plastic surgery, 51(1):23-29.

7. Pallua N, Demir E (2008): Postburn head and neck reconstruction in children with the fasciocutaneous supraclavicular artery island flap. Annals of plastic surgery, 60(3):276-282.
8. Rashid M, Zia-ul-Islam M, Bhatti AM (2006): The 'expansile'supraclavicular artery flap for release of post-burn neck contractures. Journal of Plastic, Reconstructive \& Aesthetic Surgery, 59(10):1094-1101.

9. Pallua N, Machens HG, Rennekampff O, Becker M, Berger A (1997): The fasciocutaneous supraclavicular artery island flap for releasing postburn mentosternal contractures. Plastic and reconstructive surgery, 99(7):1878-84.

10. Guerra AB, Metzinger SE, Lund KM, Cooper MM, Allen RJ, Dupin CL (2004): The thoracodorsal artery perforator flap: clinical experience and anatomic study with emphasis on harvest techniques. Plastic and reconstructive surgery, 114(1):32-41.

11. Kim YH, Lee HE, Lee JH, Kim JT, Kim SW (2017): Reliability of eccentric position of the pedicle instead of central position in a thoracodorsal artery perforator flap. Microsurgery, 37(1):4448.

12. Hamdi M, Decorte T, Demuynck M, Defrene B, Fredrickx A, Van Maele G, Monstrey S (2008): Shoulder function after harvesting a thoracodorsal artery perforator flap. Plastic and reconstructive surgery, 122(4):11111117.

13. Spinelli HM, Fink JA, Muzaffar AR (1996): The latissimus dorsi perforatorbased fasciocutaneous flap. Annals of plastic surgery, 37(5):500-506. 